\title{
ANNOTATIONS
}

\section{UNKNOWNS IN YAWS}

The first international symposium for the study of yaws control, sponsored by the World Health Organization, and held at Bangkok, Thailand, in March, 1952, was attended by representatives from 23 countries, including Belgian Congo, Brazil, Burma, Ceylon, Egypt, France, Haiti, India, Indonesia, Jamaica, Liberia, Malaya, Mexico, Netherlands, New Caledonia, Nigeria, North Borneo, Philippines, Portugal, Spain, the United Kingdom, the United States and Venezuela. The delegates from the United Kingdom were Dr. C. J. Hackett and Dr. R. R. Willcox, while Dr. S. M. Laird represented WHO from Ceylon, and Prof. K. R. Hill represented WHO from Jamaica. The proceedings of this symposium have recently been published by WHO and copies may be obtained without charge from Geneva.

The meetings were held in a hall of the Grand Palace in Bangkok, and were followed by a field trip to N.E. Thailand, in the region of Ubon, where the mass treatment campaign with penicillin was in progress. The World Health Organization is fostering a number of large-scale operations in many parts of the world : against yaws in Indonesia, Thailand, Haiti, and the Philippines, against bejel in Iraq, and against endemic syphilis in Bosnia. By the end of 1952 over $9,000,000$ persons had been examined and more than $3,000,000$ of these had been treated with penicillin. No less than $1,679,342$ of this total had been examined, and 216,047 treated, in Thailand.

The conference had the benefit of the experience of team leaders and others engaged on these operations, and particular attention was concentrated on the unknowns of this disease-of which there are still a considerable number. The nature of the organism of yaws, as far as its relationship to $T$. pallidum is concerned, is not yet fully understood. Recent work with the electron microscope and the treponemal immobilization test have not so far helped to distinguish treponemata from cases of the two clinical entities. A more thorough understanding of the causal agency will indicate how far the considerable global experience of the penicillin treatment of syphilis can be applied to yaws. While it is everywhere appreciated that transmission is usually effected by direct contact, the role, if any, of insect vectors, or even possibly of the medium of damp soil, has to be elucidated if the most economical use of preventive measures is to be evolved.

At the present time the control of yaws is being attempted by mass treatment with penicillin. Injections of 1.2 mega units procaine penicillin $G$ with aluminium monostearate, provided that the preparation is up to the standards laid down in the International Pharmacopoeia, not only give a reasonable prospect of cure for primary and secondary yaws in the individual, but, more important still, probably render the patient non-infectious. Whether a more economical dose can be used with safety is not yet certain.

The new diamine penicillin, Bicillin, introduced since the conference, will have considerable application in mass treatment programmes. "Simultaneous " treatment of an area is often a practical impossibility, and when such a task is spread over a few weeks there is a risk that the persons awaiting treatment may re-infect those already treated. By ensuring more prolonged serum levels than ever before obtained with a single injection, Bicillin may not only increase the prospects of cure in the individual but will also allow "simultaneous" treatment to become more of a reality.

Epidemiologists are aware that if these schemes are successful it will be the first time that a disease has been controlled by treatment, and they would welcome the opportunity of breaking the chain of infection at a weaker and less expensive link if such exists. Certainly no mass survey can guarantee that $\mathbf{1 0 0}$ per cent. of the population will be covered. In addition, missed cases, infectious latent cases, those in the incubation period at the time of the examination, those exposed to the disease outside the area, and their contacts-quite apart from the small proportion of relapses following treatment 
-will, if no further steps are taken, lead to a recrudescence of the disease in the community. Thus the dice is already heavily loaded against success with a single mass treatment of infectious cases alone, and it is necessary to examine and treat contacts as well, and later to revisit the area and repeat the process. What constitutes a "contact" - whether the term implies only a resident in the rondavel, or all of the companions in the kraalhas yet to be defined, as also the necessary number of re-visitations and the best interval between them.

Yaws is usually ' at the end of the road'. As countries are opened up and developed, and habits of hygiene, clothing, feeding, and living are improved, the incidence of the disease tends to decrease even in the absence of expensive mass treatment schemes. It is not known what factors are most responsible for this. Maybe single items, such as the introduction of proper clothes, the use of soap, the disuse of communal feeding bowls, the reduction of flies, or measures directed against the herding together of children, would prove to be valuable additional methods of yaws control, and would pay better ultimate dividends than mass treatment with penicillin. It is -hoped and expected, as a result of this symposium, that the considerable amount of field research necessary for the solution of these problems will be instituted, and that by the time the next symposium is held many factors will be unknowns no longer.

Meanwhile, the WHO mass treatment projects with penicillin are considered to be the most promising line of attack and are already showing encouraging immediate results. They have been likened to 'spearheads', and it is hoped that the other arms of development, medical, economic, and social, will follow in their train. 'Bridgehead' would perhaps be a better term, for 'spearheads' have a habit of being withdrawn. The consolidation phase is one for which the local health administrations will ultimately be responsible, and on this consolidation the success of these projects will ultimately stand or fall. Like dykes against the sea, the new medical defences must remain permanently manned to guard against the reappearance of the disease, or its reintroduction from outside.

It is noteworthy that the large scale mass treatment campaigns so far in operation are not taking place in British colonial territories. Whatever the reason for this British inactivity, it is certainly not that these territories lack a treponematosis problem. We may well find ourselves appearing backward to other countries, which with aided programmes are forging ahead with the control both of yaws and of the other treponematoses. If unable to embark on expensive schemes of mass treatment, we should at least develop field research in our many dependencies. Such research could be undertaken with little additional financial outlay and without employing additional staff. In each Colonial territory where yaws or other treponematoses are endemic, research should be planned to provide an answer to one particular unknown. Information is required on many points on which knowledge is at present uncertain and often lacking in detail, e.g. the precise influence of soil, rainfall, humidity, temperature, and altitude, the role of insect vectors, the important social and economic factors, the best penicillin preparation for mass treatment campaigns, and the minimum dose per patient, the most suitable type of health worker who can most economically operate such campaigns and what special training he requires for the job, the criteria for standardizing clinical diagnosis, whether all contacts should be treated and with what dosage, and the optimum time for revisiting the treated areas? The fruits of such researches would provide a rich harvest for British representatives to share at the next international symposium on the control of yaws and we hope that our unique opportunities in this field will not be neglected.

\section{REFERENCE}

"Proceedings of First International Symposium on Yaws Control." (1952). Bangkok, Thailand. WHO/VD/104. 